\title{
EVALUATION OF CERTAIN CLASSES OF EISENSTEIN-TYPE SERIES
}

\author{
HIROFUMI TSUMURA
}

(Received 28 May 2008)

\begin{abstract}
In this paper, we consider certain classes of Eisenstein-type series involving hyperbolic functions, and prove some formulas for them which can be regarded as relevant analogues of our previous results. We can also regard these formulas as certain generalizations of the famous formulas for the ordinary Eisenstein series given by Hurwitz.
\end{abstract}

2000 Mathematics subject classification: primary 11M41; secondary 11M99.

Keywords and phrases: Eisenstein series, Lemniscate constant, Riemann zeta-function.

\section{Introduction}

Let $\mathbb{N}$ be the set of natural numbers, $\mathbb{Z}$ the ring of rational integers, $\mathbb{Q}$ the field of rational numbers, $\mathbb{R}$ the field of real numbers, $\mathbb{R}_{+}$the set of positive real numbers and $\mathbb{C}$ the field of complex numbers.

Cauchy [5], Mellin [8], Ramanujan (see [3, 4]), Berndt [2] and several other mathematicians (for the details, see Berndt [2]) considered the following fascinating formulas:

$$
\begin{aligned}
\sum_{m \in \mathbb{Z} \backslash\{0\}} \frac{(-1)^{m}}{\sinh (m \pi) m^{4 k+3}} & =(2 \pi)^{4 k+3} \sum_{j=0}^{2 k+2}(-1)^{j+1} \frac{B_{2 j}(1 / 2)}{(2 j) !} \frac{B_{4 k+4-2 j}(1 / 2)}{(4 k+4-2 j) !}, \\
\sum_{m \in \mathbb{Z} \backslash\{0\}} \frac{\operatorname{coth}(m \pi)}{m^{4 k+3}} & =(2 \pi)^{4 k+3} \sum_{j=0}^{2 k+2}(-1)^{j+1} \frac{B_{2 j}(0)}{(2 j) !} \frac{B_{4 k+4-2 j}(0)}{(4 k+4-2 j) !}
\end{aligned}
$$

for $k \in \mathbb{N} \cup\{0\}$, where $\sinh x=\left(e^{x}-e^{-x}\right) / 2, \quad \cosh x=\left(e^{x}+e^{-x}\right) / 2, \quad \operatorname{coth} x=$ $\cosh x / \sinh x$ and $B_{n}(x)$ is the $n$th Bernoulli polynomial defined by

$$
\frac{t e^{x t}}{e^{t}-1}=\sum_{N=0}^{\infty} B_{N}(x) \frac{t^{N}}{N !} .
$$

(C) 2009 Australian Mathematical Society 0004-9727/2009 \$16.00 
In particular, it is known that the formula (1.2) can be derived directly from the famous formula given by Ramanujan:

$$
\begin{aligned}
\alpha^{-N} & \left\{\frac{1}{2} \zeta(2 N+1)+\sum_{m=1}^{\infty} \frac{1}{\left(e^{2 m \alpha}-1\right) m^{2 N+1}}\right\} \\
= & (-\beta)^{-N}\left\{\frac{1}{2} \zeta(2 N+1)+\sum_{m=1}^{\infty} \frac{1}{\left(e^{2 m \beta}-1\right) m^{2 N+1}}\right\} \\
& -2^{2 N} \sum_{m=0}^{N+1}(-1)^{m} \frac{B_{2 m}(0)}{(2 m) !} \frac{B_{2 N+2-2 m}(0)}{(2 N+2-2 m) !} \alpha^{N+1-m} \beta^{m},
\end{aligned}
$$

where $N$ is any nonzero integer, $\alpha$ and $\beta$ are positive numbers such that $\alpha \beta=\pi^{2}$ and $\zeta(s)$ is the Riemann zeta-function (see Ramanujan's notebooks [3, (25.3), p. 293]).

Recently, in our previous paper [10], we studied certain Eisenstein-type analogues of (1.1) defined by

$$
\begin{aligned}
& \mathcal{G}_{k}(i)=\sum_{n \in \mathbb{Z}}(-1)^{n} \sum_{m \in \mathbb{Z} \backslash\{0\}} \frac{1}{\sinh (m \pi)(m+n i)^{k}} \quad(k \in \mathbb{N}), \\
& \mathcal{H}_{k}(i)=\sum_{n \in \mathbb{Z}}(-1)^{n} \sum_{m \in \mathbb{Z} \backslash\{0\}} \frac{1}{\cosh (m \pi)(m+n i)^{k}} \quad(k \in \mathbb{N}) .
\end{aligned}
$$

Note that if $k \geq 2$, then these series are absolutely convergent. Furthermore, even if $k=1$, then these series are convergent. In order to evaluate these values, we recall that the Eisenstein series $G_{4 j}(i)$ can be expressed as

$$
G_{4 j}(i)=\sum_{\substack{m, n \in \mathbb{Z} \\(m, n) \neq(0,0)}} \frac{1}{(m+n i)^{4 j}}=\frac{(2 \varpi)^{4 j}}{(2 j) !} H_{4 j} \quad(j \in \mathbb{N})
$$

for $i=\sqrt{-1}$ and

$$
\varpi=2 \int_{0}^{1} \frac{d x}{\sqrt{1-x^{4}}}=2.622057 \ldots,
$$

where $\left\{H_{4 m} \mid m \in \mathbb{N}\right\}$ are called the Hurwitz numbers defined as coefficients of the Laurent series expansion of the Weierstrass $\mathfrak{p}$-function, namely,

$$
H_{4}=\frac{1}{10}, \quad H_{8}=\frac{3}{10}, \quad H_{12}=\frac{567}{130}, \quad H_{16}=\frac{43659}{170}, \ldots
$$


(see [6]; also [1, 7]). By using these facts, we evaluated $\mathcal{G}_{2 k+1}(i)$ and $\mathcal{H}_{2 k+2}(i)$ $(k \in \mathbb{N} \cup\{0\})$ explicitly in terms of $\pi$ and $\varpi$. For example, we gave

$$
\begin{aligned}
\mathcal{G}_{7}(i) & =\sum_{\substack{m, n \in \mathbb{Z} \\
m \neq 0}} \frac{(-1)^{n}}{\sinh (m \pi)(m+n i)^{7}} \\
& =\frac{1}{525} \frac{\varpi^{8}}{\pi}+\frac{7}{5400} \varpi^{4} \pi^{3}-\frac{127}{75600} \pi^{7}+\frac{31}{15120} \pi^{6}, \\
\mathcal{H}_{4}(i) & =\sum_{\substack{m, n \in \mathbb{Z} \\
m \neq 0}} \frac{(-1)^{n}}{\cosh (m \pi)(m+n i)^{4}}=\frac{1}{15} \varpi^{4}-\frac{17}{90} \pi^{4}+\frac{1}{2} \pi^{3}
\end{aligned}
$$

(see [10, Examples 4.1 and 4.2]).

In this paper, with the aim to giving Eisenstein-type analogues of Ramanujan's formula (1.2), we consider

$$
\mathfrak{C}_{k}^{v}(i)=\sum_{\substack{m, n \in \mathbb{Z} \\ m \neq 0}} \frac{(\operatorname{coth}(m \pi))^{v}}{(m+n i)^{k}}
$$

for $k \in \mathbb{N}$ with $k \geq 3$ and $v \in \mathbb{Z}$. Note that

$$
\mathfrak{C}_{4 j}^{0}(i)=G_{4 j}(i)-2 \zeta(4 j) \quad(j \in \mathbb{N}) .
$$

By the same method as introduced in [10], we prove that

$$
\mathfrak{C}_{k}^{v}(i)=\sum_{\substack{m, n \in \mathbb{Z} \\ m \neq 0}} \frac{(\operatorname{coth}(m \pi))^{v}}{(m+n i)^{k}} \in \mathbb{Q}\left[\frac{1}{\pi}, \pi, \varpi^{4}\right]
$$

for $k \geq 3$ and $v \in \mathbb{N} \cup\{0\}$ with $k \equiv v(\bmod 2)$ (see Corollary 2.5). Actually, when $k, l \in \mathbb{Z}$ with $k \geq 3, l \geq 0$ and $k \equiv l(\bmod 2)$, we can express $\mathfrak{C}_{k}^{l}(i)$ as a closed form in terms of $\varpi$ and $\pi$ (see Example 2.6). Note that if $k \not \equiv l(\bmod 2)$, then $\mathfrak{C}_{k}^{l}(i)=0$. In particular, (1.11) in the case of $(k, v)=(4 j, 0)$ essentially implies (1.5) by (1.10).

For example, as analogues of Ramanujan's formula (1.2), we obtain

$$
\begin{aligned}
& \mathfrak{C}_{3}^{1}(i)=\sum_{\substack{m, n \in \mathbb{Z} \\
m \neq 0}} \frac{\operatorname{coth}(m \pi)}{(m+n i)^{3}}=\frac{\varpi^{4}}{15 \pi}+\frac{4}{45} \pi^{3}-\frac{1}{3} \pi^{2}, \\
& \mathfrak{C}_{4}^{2}(i)=\sum_{\substack{m, n \in \mathbb{Z} \\
m \neq 0}} \frac{(\operatorname{coth}(m \pi))^{2}}{(m+n i)^{4}}=\frac{2}{45} \varpi^{4}+\frac{16}{945} \pi^{4}-\frac{4}{45} \pi^{3}
\end{aligned}
$$

(see Example 2.6). 


\section{Inductive relation formulas}

As a generalization of $\mathcal{G}_{k}(i)$ and $\mathcal{H}_{k}(i)$, we define

$$
\mathcal{G}_{k}^{l}(i)=\sum_{n \in \mathbb{Z}}(-1)^{n} \sum_{m \in \mathbb{Z} \backslash\{0\}} \frac{(\operatorname{coth}(m \pi))^{l}}{\sinh (m \pi)(m+n i)^{k}}
$$

for $k \in \mathbb{N}$ and $l \in \mathbb{Z}$. Note that $\mathcal{G}_{k}^{0}(i)=\mathcal{G}_{k}(i)$ and $\mathcal{G}_{k}^{-1}(i)=\mathcal{H}_{k}(i)$. We prove the following theorem which is essentially the main result in this paper.

Theorem 2.1. For $p \in \mathbb{N}$ and $l \in \mathbb{Z}$,

$$
\begin{aligned}
& \mathfrak{C}_{2 p+1}^{2 l+1}(i)=-\frac{2(-1)^{p}}{\pi} \sum_{\tau=1}^{p} \zeta(2 p-2 \tau)(-1)^{\tau} \mathfrak{C}_{2 \tau+2}^{2 l}(i)-\frac{2(-1)^{p}}{\pi^{2}} \zeta(2 p) \mathfrak{C}_{3}^{2 l-1}(i), \\
& \mathfrak{C}_{2 p+2}^{2 l}(i)=-\frac{2(-1)^{p}}{\pi} \sum_{\tau=0}^{p} \zeta(2 p-2 \tau)(-1)^{\tau} \mathfrak{C}_{2 \tau+3}^{2 l-1}(i) .
\end{aligned}
$$

In order to prove this theorem, we first prepare an analogous result of our previous result.

LEMMA 2.2. For $k \in \mathbb{N}$ and $\theta \in(-\pi, \pi)$,

$$
\begin{aligned}
\frac{1}{2} & \sum_{m \in \mathbb{Z} \backslash\{0\}} \sum_{n \in \mathbb{Z}} \frac{(-1)^{n}(\operatorname{coth}(m \pi))^{v}\left\{e^{(m+n i) \theta}-e^{-(m+n i) \theta}\right\}}{\sinh (m \pi)(m+n i)^{k+2}} \\
= & \sum_{j=0}^{[k / 2]} \mathcal{G}_{k+1-2 j}^{v}(i) \frac{\theta^{2 j+1}}{(2 j+1) !} .
\end{aligned}
$$

PROOF. We only have to use the same method as in the proof of in [10, (3.7)]. Then we can similarly obtain (2.4), so we omit the proof here.

Note that both sides of (2.4) are continuous for $\theta \in[-\pi, \pi]$. Hence, letting $\theta \rightarrow \pi$ and using $\left(e^{(m+n i) \pi}-e^{-(m+n i) \pi}\right) / 2=\sinh (m \pi)(-1)^{n}$, we can confirm that

$$
\mathfrak{C}_{k+2}^{v}(i)=\sum_{\substack{m, n \in \mathbb{Z} \\ m \neq 0}} \frac{(\operatorname{coth}(m \pi))^{v}}{(m+n i)^{k+2}}=\sum_{j=0}^{[k / 2]} \mathcal{G}_{k+1-2 j}^{v}(i) \frac{\pi^{2 j+1}}{(2 j+1) !}
$$

Similarly, we can prove

$$
\frac{1}{2} \sum_{m \in \mathbb{Z} \backslash\{0\}} \sum_{n \in \mathbb{Z}} \frac{(-1)^{n}(\operatorname{coth}(m \pi))^{v}\left\{e^{(m+n i) \theta}+e^{-(m+n i) \theta}\right\}}{\sinh (m \pi)(m+n i)^{k+1}}=\sum_{j=0}^{[k / 2]} \mathcal{G}_{k+1-2 j}^{v}(i) \frac{\theta^{2 j}}{(2 j) !}
$$


Since $\operatorname{coth} x=\cosh x / \sinh x$, letting $\theta \rightarrow \pi$, we have

$$
\mathfrak{C}_{k+1}^{v+1}(i)=\sum_{\substack{m, n \in \mathbb{Z} \\ m \neq 0}} \frac{(\operatorname{coth}(m \pi))^{v+1}}{(m+n i)^{k+1}}=\sum_{j=0}^{[k / 2]} \mathcal{G}_{k+1-2 j}^{v}(i) \frac{\pi^{2 j}}{(2 j) !}
$$

for $k \in \mathbb{N}$ with $k \geq 2$.

Put $k=2 p+\mu$ for $p \in \mathbb{N}$ and $\mu \in\{0,1\}$ in (2.5) and (2.7). Then

$$
\mathfrak{C}_{2 p+\mu+2}^{v}(i)=\sum_{j=0}^{p} \mathcal{G}_{2 p+\mu+1-2 j}^{v}(i) \frac{\pi^{2 j+1}}{(2 j+1) !}
$$

and

$$
\mathfrak{C}_{2 p+\mu+1}^{v+1}(i)=\sum_{j=0}^{p} \mathcal{G}_{2 p+\mu+1-2 j}^{v}(i) \frac{\pi^{2 j}}{(2 j) !} .
$$

Note that (2.8) also holds for $p=0$ if $\mu=1$, because (2.5) holds for $k=1$. Now we recall the following lemma.

LeMma 2.3 [9, Lemma 4.4]. Let $\left\{P_{2 h}\right\},\left\{Q_{2 h}\right\},\left\{R_{2 h}\right\}$ be sequences such that

$$
P_{2 h}=\sum_{j=0}^{h} R_{2 h-2 j} \frac{(i \pi)^{2 j}}{(2 j) !}, \quad Q_{2 h}=\sum_{j=0}^{h} R_{2 h-2 j} \frac{(i \pi)^{2 j}}{(2 j+1) !}
$$

for any $h \in \mathbb{N} \cup\{0\}$. Then

$$
P_{2 h}=-2 \sum_{\tau=0}^{h} \zeta(2 h-2 \tau) Q_{2 \tau}
$$

for any $h \in \mathbb{N} \cup\{0\}$.

In order to apply this lemma, we transform (2.8) and (2.9) into

$$
\frac{(-1)^{p}}{\pi} \mathfrak{C}_{2 p+\mu+2}^{v}(i)=\sum_{j=0}^{p}(-1)^{p-j} \mathcal{G}_{2 p-2 j+\mu+1}^{v}(i) \frac{(i \pi)^{2 j}}{(2 j+1) !}
$$

and

$$
(-1)^{p} \mathfrak{C}_{2 p+\mu+1}^{v+1}(i)=\sum_{j=0}^{p}(-1)^{p-j} \mathcal{G}_{2 p-2 j+\mu+1}^{v}(i) \frac{(i \pi)^{2 j}}{(2 j) !} .
$$

In Lemma 2.3, we put $P_{0}=Q_{0}=R_{0}=\mathcal{G}_{\mu+1}^{v}(i)$ and

$$
P_{2 h}=(-1)^{h} \mathfrak{C}_{2 h+\mu+1}^{v+1}(i), \quad Q_{2 h}=\frac{(-1)^{h}}{\pi} \mathfrak{C}_{2 h+\mu+2}^{v}(i), \quad R_{2 h}=(-1)^{h} \mathcal{G}_{2 h+\mu+1}^{v}(i)
$$


for $h \in \mathbb{N}$. Then it follows from (2.11) that

$$
(-1)^{p} \mathfrak{C}_{2 p+\mu+1}^{v+1}(i)=-2 \sum_{\tau=1}^{p} \zeta(2 p-2 \tau) \frac{(-1)^{\tau}}{\pi} \mathfrak{C}_{2 \tau+\mu+2}^{v}(i)-2 \zeta(2 p) \mathcal{G}_{\mu+1}^{v}(i)
$$

for $p \in \mathbb{N}$.

Next we determine $\mathcal{G}_{\mu+1}^{\nu}(i)$ for $\mu=0,1$. As noted above, (2.12) holds for $p=0$ when $\mu=1$. Hence, we can determine the case $\mu=1$, namely

$$
\mathcal{G}_{2}^{v}(i)=\frac{1}{\pi} \mathfrak{C}_{3}^{v}(i) \quad(v \in \mathbb{Z})
$$

Furthermore, in order to consider the case $\mu=0$, we need the following lemma.

LEMMA 2.4. For $v \in \mathbb{Z}, \pi \mathcal{G}_{1}^{v}(i)=\mathcal{G}_{2}^{v-1}(i)$.

Proof. From the definition (2.1), we can see that $\mathcal{G}_{k}^{v}(i)=0$ when $k \equiv v(\bmod 2)$ by changing the summation indices $(m, n)$ into $(-m,-n)$. Hence, if $v$ is odd, we trivially obtain the assertion. So we assume that $v$ is even. If $\theta \in(-\pi, \pi)$, we see that the left-hand side of (2.4) in the case $k=0$ is absolutely convergent. Hence,

$$
\begin{aligned}
\frac{1}{2} & \sum_{m \in \mathbb{Z} \backslash\{0\}} \sum_{n \in \mathbb{Z}} \frac{(-1)^{n}(\operatorname{coth}(m \pi))^{v}\left\{e^{(m+n i) \theta}-e^{-(m+n i) \theta}\right\}}{\sinh (m \pi)(m+n i)^{2}} \\
& =\sum_{m=1}^{\infty} \sum_{n \in \mathbb{Z}} \frac{(-1)^{n}(\operatorname{coth}(m \pi))^{v}\left\{e^{(m+n i) \theta}-e^{-(m+n i) \theta}\right\}}{\sinh (m \pi)(m+n i)^{2}} \\
& =\mathcal{G}_{1}^{v}(i) \theta \quad(\theta \in(-\pi, \pi)),
\end{aligned}
$$

because $v$ is even. On the left-hand side of (2.16), we consider $\theta=\pi+(\theta-\pi)$, namely

$$
e^{(m+n i)(\pi+\theta-\pi)}=(-1)^{n} e^{m \pi} e^{(m+n i)(\theta-\pi)} .
$$

Then the left-hand side of (2.16) can be written as

$$
\begin{aligned}
\sum_{m=1}^{\infty} & \sum_{n \in \mathbb{Z}} \frac{(\operatorname{coth}(m \pi))^{v}\left\{e^{m \pi} e^{(m+n i)(\theta-\pi)}-e^{-m \pi} e^{-(m+n i)(\theta-\pi)}\right\}}{\sinh (m \pi)(m+n i)^{2}} \\
= & \sum_{m=1}^{\infty} \sum_{n \in \mathbb{Z}} \frac{(\operatorname{coth}(m \pi))^{v}\left\{\left(e^{m \pi}-e^{-m \pi}\right) e^{(m+n i)(\theta-\pi)}\right\}}{\sinh (m \pi)(m+n i)^{2}} \\
& +\sum_{m=1}^{\infty} \sum_{n \in \mathbb{Z}} \frac{(\operatorname{coth}(m \pi))^{v}\left\{e^{-m \pi}\left(e^{(m+n i)(\theta-\pi)}-e^{-(m+n i)(\theta-\pi)}\right)\right\}}{\sinh (m \pi)(m+n i)^{2}} \\
= & 2 \sum_{m=1}^{\infty} \sum_{n \in \mathbb{Z}} \frac{(\operatorname{coth}(m \pi))^{v} e^{(m+n i)(\theta-\pi)}}{(m+n i)^{2}} \\
& +\sum_{m=1}^{\infty} \sum_{n \in \mathbb{Z}} \frac{(\operatorname{coth}(m \pi))^{v} e^{-m \pi}\left\{e^{(m+n i)(\theta-\pi)}-e^{-(m+n i)(\theta-\pi)}\right\}}{\sinh (m \pi)(m+n i)^{2}} .
\end{aligned}
$$


For simplicity, we denote by $\xi^{\nu}(\theta)$ and $\eta^{\nu}(\theta)$ the first term and the second term on the right-hand side of (2.17), respectively. Then (2.16) can be rewritten as

$$
\xi^{\nu}(\theta)+\eta^{v}(\theta)=\mathcal{G}_{1}^{v}(i) \theta \quad(\theta \in(-\pi, \pi)) .
$$

Furthermore, we similarly consider the left-hand side of (2.6) in the case $k=1$ by replacing $v$ by $v-1$, and by putting $\theta=\pi+(\theta-\pi)$. Then, in the same way, we have

$$
\xi^{\nu}(\theta)-\eta^{\nu-1}(\theta)=\mathcal{G}_{2}^{\nu-1}(i) \quad(\theta \in(-\pi, \pi)) .
$$

Combining (2.18) and (2.19), we have

$$
\mathcal{G}_{1}^{\nu}(i) \theta-\eta^{\nu}(\theta)=\mathcal{G}_{2}^{\nu-1}(i)+\eta^{\nu-1}(\theta) \quad(\theta \in(-\pi, \pi)) .
$$

Note that if $\theta \in[\pi / 2, \pi]$, then $\eta^{v}(\theta)$ is absolutely and uniformly convergent with respect to $\theta$. Hence, we have

$$
\lim _{\theta \rightarrow \pi} \eta^{v}(\theta)=\eta^{v}(\pi)=0 \quad(\nu \in \mathbb{Z}) .
$$

Therefore, by letting $\theta \rightarrow \pi$ in (2.20), we have the assertion.

Proof of Theorem 2.1. Combining (2.15) and Lemma 2.4, we have

$$
\mathcal{G}_{1}^{\nu}(i)=\frac{1}{\pi} \mathcal{G}_{2}^{\nu-1}(i)=\frac{1}{\pi^{2}} \mathfrak{C}_{3}^{\nu-1}(i) \quad(v \in \mathbb{Z}) .
$$

Hence, combining (2.14) and (2.21), we have the proof of Theorem 2.1.

From Theorem 2.1, we can inductively evaluate $\mathfrak{C}_{k}^{l}(i)$ for $k \geq 3, l \in \mathbb{N} \cup\{0\}$ with $k \equiv l(\bmod 2)$. First we check the case $l=0$ in (2.2) and (2.3). In [10, Example 4.1], we have already proved that

$$
\frac{1}{\pi^{2}} \mathfrak{C}_{3}^{-1}(i)=\mathcal{G}_{1}^{0}(i)=-1+\frac{\pi}{3}
$$

using (2.21). Moreover, we see that $\mathfrak{C}_{2 j}^{0}(i)=G_{2 j}(i)-2(-1)^{j} \zeta(2 j)$ for $j \geq 2$, where $G_{2 j}(i)$ is the Eisenstein series. Note that $G_{4 j}(i)$ can be evaluated in terms of $\varpi$ (see $(1.5)$ ) and $G_{4 j+2}(i)=0$ for $j \in \mathbb{N}$. Hence, we have $\mathfrak{C}_{2 j}^{0}(i) \in \mathbb{Q}\left[\pi, \varpi^{4}\right]$ for $j \geq 2$. Therefore, by (2.2) and (2.22), we can see that $\mathfrak{C}_{2 p+1}^{1}(i) \in \mathbb{Q}\left[1 / \pi, \pi, \varpi^{4}\right]$ for $p \in \mathbb{N}$. Furthermore, using (2.2) and (2.3) alternately, we can easily prove the following corollary by induction.

Corollary 2.5. For $k, v \in \mathbb{Z}$ with $k \geq 3, v \geq-1$ and $k \equiv v(\bmod 2)$,

$$
\mathfrak{C}_{k}^{v}(i)=\sum_{\substack{m, n \in \mathbb{Z} \\ m \neq 0}} \frac{(\operatorname{coth}(m \pi))^{v}}{(m+n i)^{k}} \in \mathbb{Q}\left[\frac{1}{\pi}, \pi, \varpi^{4}\right] .
$$


EXAMPLE 2.6. By using (2.2) and (2.3), we can inductively obtain the following concrete examples of Corollary 2.5 , which are numerically correct:

$$
\begin{aligned}
& \mathfrak{C}_{3}^{1}(i)=\sum_{\substack{m, n \in \mathbb{Z} \\
m \neq 0}} \frac{\operatorname{coth}(m \pi)}{(m+n i)^{3}}=\frac{\varpi^{4}}{15 \pi}+\frac{4}{45} \pi^{3}-\frac{1}{3} \pi^{2}, \\
& \mathfrak{C}_{4}^{2}(i)=\sum_{\substack{m, n \in \mathbb{Z} \\
m \neq 0}} \frac{(\operatorname{coth}(m \pi))^{2}}{(m+n i)^{4}}=\frac{2}{45} \varpi^{4}+\frac{16}{945} \pi^{4}-\frac{4}{45} \pi^{3}, \\
& \mathfrak{C}_{5}^{5}(i)=\sum_{m, n \in \mathbb{Z}} \frac{(\operatorname{coth}(m \pi))^{5}}{(m+n i)^{5}}=\frac{\varpi^{8}}{315 \pi^{3}}+\frac{46}{2835} \varpi^{4} \pi+\frac{52}{31185} \pi^{5}-\frac{1}{63} \pi^{4}, \\
& \mathfrak{C}_{6}^{4}(i)=\sum_{\substack{m, n \in \mathbb{Z} \\
m \neq 0}} \frac{(\operatorname{coth}(m \pi))^{4}}{(m+n i)^{6}}=\frac{4 \varpi^{8}}{1575 \pi^{2}}+\frac{62}{14175} \varpi^{4} \pi^{2}-\frac{1024}{467775} \pi^{6}+\frac{8}{1575} \pi^{5}, \\
& \mathfrak{C}_{7}^{3}(i)=\sum_{\substack{m, n \in \mathbb{Z} \\
m \neq 0}} \frac{(\operatorname{coth}(m \pi))^{3}}{(m+n i)^{7}}=\frac{\varpi^{8}}{525 \pi}-\frac{1}{14175} \varpi^{4} \pi^{3}-\frac{136}{155925} \pi^{7}+\frac{2}{525} \pi^{6} .
\end{aligned}
$$

Combining (2.5), (2.7), (2.21) and Corollary 2.5, we can obtain the following result which is a certain generalization of our previous result in [10] because $\mathcal{G}_{k}^{0}(i)=\mathcal{G}_{k}(i)$ and $\mathcal{G}_{k}^{-1}(i)=\mathcal{H}_{k}(i)$.

Corollary 2.7. For $k \in \mathbb{N}, v \in \mathbb{Z}$ with $v \geq-1$ and $k \not \equiv v(\bmod 2)$,

$$
\mathcal{G}_{k}^{v}(i)=\sum_{n \in \mathbb{Z}}(-1)^{n} \sum_{\substack{m \in \mathbb{Z} \\ m \neq 0}} \frac{(\operatorname{coth}(m \pi))^{v}}{\sinh (m \pi)(m+n i)^{k}} \in \mathbb{Q}\left[\frac{1}{\pi}, \pi, \varpi^{4}\right] .
$$

ExAmple 2.8. Combining (2.5), (2.7), (2.21) and Theorem 2.1, for example, we can obtain the concrete examples of Corollary 2.7 :

$$
\begin{aligned}
& \mathcal{G}_{2}^{1}(i)=\frac{\varpi^{4}}{15 \pi^{2}}+\frac{4}{45} \pi^{2}-\frac{1}{3} \pi, \\
& \mathcal{G}_{2}^{3}(i)=\frac{\varpi^{4}}{15 \pi^{2}}+\frac{44}{945} \pi^{2}-\frac{1}{5} \pi, \\
& \mathcal{G}_{3}^{2}(i)=\frac{\varpi^{4}}{30 \pi}+\frac{2}{945} \pi^{3}-\frac{1}{30} \pi^{2}, \\
& \mathcal{G}_{4}^{1}(i)=\frac{1}{90} \varpi^{4}-\frac{26}{945} \pi^{4}+\frac{7}{90} \pi^{3}, \\
& \mathcal{G}_{4}^{5}(i)=\frac{\varpi^{8}}{350 \pi^{4}}+\frac{83}{14175} \varpi^{4}-\frac{1574}{467775} \pi^{4}+\frac{1}{126} \pi^{3}, \\
& \mathcal{G}_{5}^{4}(i)=\frac{\varpi^{8}}{450 \pi^{3}}-\frac{113}{113400} \varpi^{4} \pi-\frac{1163}{311850} \pi^{5}+\frac{1}{72} \pi^{4} .
\end{aligned}
$$




\section{Acknowledgements}

The author would like to express his sincere gratitude to Professors Kohji Matsumoto and Yasushi Komori for their valuable comments and precise suggestions.

\section{References}

[1] R. Ayoub, 'Euler and the zeta function', Amer. Math. Monthly 81 (1974), 1067-1086.

[2] B. C. Berndt, 'Analytic Eisenstein series, theta-functions, and series relations in the spirit of Ramanujan', J. Reine Angew Math. 303/304 (1978), 332-365.

[3] - Ramanujan's Notebooks, part II (Springer, New York, 1989).

[4] _ Ramanujan's Notebooks, part V (Springer, New York, 1998).

[5] A. Cauchy, Oeuvres Complètes D'Augustin Cauchy, Série II, Tome VII (Gauthier-Villars, Paris, 1889).

[6] A. Hurwitz, 'Mathematische Werke. Bd. I: Funktionentheorie', in: Herausgegeben von der Abteilung für Mathematik und Physik der Eidgenössischen Technischen Hochschule in Zürich (Birkhäuser, Basel, 1962).

[7] F. Lemmermeyer, Reciprocity Laws: From Euler to Eisenstein (Springer, Berlin, 2000).

[8] H. Mellin, 'Eine Formel für den Logarithmus transcendenter Funktionen von endlichen Geschlecht', Acta Soc. Sci. Fennicae 29 (1902), 165-183.

[9] H. Tsumura, 'On functional relations between the Mordell-Tornheim double zeta functions and the Riemann zeta function', Math. Proc. Cambridge Philos. Soc. 142 (2007), 395-405.

[10] - 'On certain analogues of Eisenstein series and their evaluation formulas of Hurwitz type', Bull. London Math. Soc. 40 (2008), 85-93.

HIROFUMI TSUMURA, Department of Mathematics and Information Sciences, Tokyo Metropolitan University, 1-1, Minami-Ohsawa, Hachioji, Tokyo 192-0397, Japan

e-mail: tsumura@tmu.ac.jp 